University of Nebraska - Lincoln

DigitalCommons@University of Nebraska - Lincoln

Gordon Gallup Publications

Research Papers in Physics and Astronomy

February 1968

\title{
Projected Hartree Product Wavefunctions
}

Gordon A. Gallup

UNL,ggallup1@unl.edu

Follow this and additional works at: https://digitalcommons.unl.edu/physicsgallup

Part of the Physics Commons

Gallup, Gordon A., "Projected Hartree Product Wavefunctions" (1968). Gordon Gallup Publications. 23. https://digitalcommons.unl.edu/physicsgallup/23

This Article is brought to you for free and open access by the Research Papers in Physics and Astronomy at DigitalCommons@University of Nebraska - Lincoln. It has been accepted for inclusion in Gordon Gallup Publications by an authorized administrator of DigitalCommons@University of Nebraska - Lincoln. 


\title{
Projected Hartree Product Wavefunctions*
}

\author{
G. A. Gallup \\ Department of Chemistry, University of Nebraska, Lincoln, Nebraska
}

(Received 12 July 1967)

\begin{abstract}
A method for performing a restricted configuration interaction calculation on atoms or molecules based on the use of Young operators from the symmetric groups is discussed and its relation to some other types of calculations is pointed out. The method in its most general form can be said to be an extension of the independent particle approach which represents the wavefunction in terms of the $n$ best possible oneparticle orbitals for the $n$-particle system. Ilustrations are given for the hydrogen molecule, the helium atom, and the allyl radical.
\end{abstract}

\section{INTRODUCTION}

In some of his early work on the helium atom, Hylleraas $^{1}$ investigated the best wavefunction of the form

$$
\Psi=\exp \left(-\alpha_{1} r_{1}-\alpha_{2} r_{2}\right)+\exp \left(-\alpha_{2} r_{1}-\alpha_{1} r_{2}\right),
$$

by minimization with respect to the variational parameters $\alpha_{1}$ and $\alpha_{2}$. This "split orbital" function is one of the earliest approximate examples of a general method proposed by Löwdin ${ }^{2}$ and called by him the projected Hartree-Fock (PHF) method. Very briefly, Löwdin suggests the construction of a sequence of commuting projection operators $F_{1}, F_{2}, \cdots, F_{i}$ for specific eigenvalues of each of the constants of the motion, and the minimization of

$$
W=\left\langle\Psi\left|H F_{1} F_{2} \cdots F_{i}\right| \Psi\right\rangle /\left\langle\Psi\left|F_{1} F_{2} \cdots F_{i}\right| \Psi\right\rangle
$$

with respect to $|\Psi\rangle$ of the Slater determinantal type. Löwdin has emphasized that (PHF) is really an extension of the independent particle model rather than a type of configuration interaction method, although the details of computation are very similar. It is evident that functions minimizing $W$ in Eq. (2) are not unique since the metric $F_{1} \cdots F_{i}$ is not a positive definite operator, but possesses a null space. Nevertheless, as we shall see later, suitable additional conditions may be used in the present context to remove the uncertainty without destroying the generality of the method.

More recently Matsen $\mathrm{et} \mathrm{al.}{ }^{3}$ have given an extensive discussion of the use of representation theory of symmetric groups for the construction of what is essentially the spatial part of an antisymmetrized eigenfunction of the total spin angular momentum operator. It seems of interest to fuse these two ideas, and instead of using

* This work was done in part under the auspices of the University of Nebraska Research Council.

$\uparrow$ On leave during academic year 1967-1968 at Quantum Theory Project, Nuclear Sciences Building, University of Florida, Gainesville, Fla.

1 E. A. Hylleraas, Z. Physik 54, 347 (1929).

2 P.-O. Lowdin, Phys. Rev. 97, 1509 (1955); see also article in Quantum Theory of Atoms, Molecules and the Solid State (Academic Press Inc., New York, 1966).

3 (a) F. A. Matsen, Advan. Quantum Chem. 1, 59f (1964).

(b) F. A. Matsen, J. Phys. Chem. 68, 3282 (1964); 70, 1568

(1966). (c) F. A. Matsen, A. A. Cantu, and R. D. Poshusta, ibid. 70, 1558 (1966).
Slater determinantal functions in Eq. (2), we may return to the original form of self-consistant field theory proposed by Hartree ${ }^{4}$ and use a product function in Eq. (2). This seems particularly attractive for molecular problems in the spin-free approximation since there is one group projection necessary, the cross product between the groups for spatial symmetry and exchange symmetry.

Goddard also ${ }^{5}$ has discussed the symmetric groups in defining projection operators which produce an antisymmetric eigenfunction of the total spin and uses these operators to define a set of Hartree-Fock-like equations. His operators are based on Young's orthogonal representation of the symmetric group.

This article discusses some aspects of this problem and gives applications to the helium atom and the allyl radical.

\section{CHARACTERISTIC UNITS OF THE FROBENIUS ALGEBRA OF A GROUP}

Before examining the quantum mechanical problem, it will be useful to investigate some purely group theoretical subjects.

In his pioneering work on the representation theory of groups, Frobenius introduced the concept of a group algebra. ${ }^{6}$ By analysis of this group algebra it is possible to show that a set of elements of the algebra exist called primitive characteristic units that have the property,

$$
e_{j j^{(i)}} e_{j^{\prime} j^{\prime}}{ }^{\left(i^{\prime \prime}\right)}=e_{j j^{(i)}}{ }^{\left(i i^{\prime}\right.} \delta_{j j^{\prime}} \text {. }
$$

Wigner has shown that these characteristic units may be written explicitely in terms of elements of irreducible representation matrices of the group. If we denote the $i$ th irreducible representation by $D^{(i)}(R)$ and its dimension by $f_{i}$,

$$
e_{j j}^{(i)}=\left(f_{i} / g\right) \sum_{R} D^{(i)}\left(R^{-1}\right)_{j j} R,
$$

${ }^{4}$ D. R. Hartree, Proc. Cambridge Phil. Soc. 24, 89 (1928).

5 W. A. Goddard, Phys. Rev. 157, 73, 81, 93 (1967).

${ }^{6}$ See, e.g., D. E. Littlewood, Theory of Group Characters (Oxford University Press, London, Eng., 1940), Chapter 2.

7 E. P. Wigner, Group Theory and Its Application to the Quantum Mechanics of Atomic Spectra (Academic Press Inc., New York, 1959), Chap. 12. 
where the sum is taken over all elements of the group and $g$ is the order of the group. The off-diagonal elements of $D^{(i)}(R)$ produce elements of the algebra which are also useful, i.e.,

$$
e_{j k}^{(i)}=\left(f_{i} / g\right) \sum_{R} D^{(i)}\left(R^{-1}\right)_{k j} R
$$

These elements have the property

$$
e_{j k}^{(i)} e_{i m}^{\left(i^{\prime}\right)}=e_{j m}{ }^{(i)} \delta_{i i^{\prime}} \delta_{k l l},
$$

and it is seen that Eq. (6) includes the result in Eq. (3). The elements of the algebra defined in Eq. (5) have the typical shifting operator property, since it is easily shown that

$$
e_{j k}^{(i)} e_{k k}{ }^{(i)} e_{k j}^{(i)}=e_{j j}{ }^{(i)} .
$$

Löwdin ${ }^{8}$ has recently given a very thorough discussion of group algebra.

In our later work the elements of the group, $R$, will be considered linear operators in the Hilbert space of our problem and it is easily seen that they are unitary operators, $R^{-1}=R^{\dagger}$. The most useful type of characteristic unit for our purposes is Hermitian and if $D^{(i)}$ is a unitary representation of the group $e_{j k}{ }^{(i)}$ has the property

$$
e_{j k}^{(i)}=e_{k j}^{(i) \dagger},
$$

and hence $e_{j j}{ }^{(i)}$ is Hermitian. The converse theorem is easily proved that if, for example, $e_{11}{ }^{(i)}$ is Hermitian, there is a unitary $D^{(i)}$ upon which it may be based. We are able to say, therefore, that any characteristic unit of the Frobenius algebra which is Hermitian has coefficients which are diagonal elements of an irreducible unitary representation of the group. Thus if we have

$$
\begin{aligned}
e^{(i)} & =\left(f_{i} / g\right) \sum_{R} a_{R} R, \\
e^{(i)} & =e^{(i) \dagger}, \\
e^{(i) 2} & =e^{(i)} \\
a_{R^{-1}} & =a_{R}{ }^{*}, \\
a_{I} & =1,
\end{aligned}
$$

there is a unitary representation such that

$$
D^{(i)}(R)_{11}=a_{R}^{*} .
$$

Equation (8) forces a restriction on the magnitude of the coefficients, viz., $\left|a_{R}\right| \leq 1$.

For later purposes we wish to define the trace of $e^{(i)}$ with respect to a given basis. If we have a vector space $V$ which affords a representation of the group, then one obtains

$$
R: v \rightarrow v^{\prime}=D_{V}(R) v ; \quad v \in V .
$$

The characters of $D_{V}(R)$ will be denoted $\chi_{V}(R)$ for the element $R$ and $\chi_{V}(\rho)$ for the elements in the $\rho$ th

\footnotetext{
${ }^{8}$ P.-O. Löwdin, Rev. Mod. Phys. 39, 259 (1967).
}

class. We define

$$
\begin{aligned}
t_{V}^{(i)} & =\operatorname{Tr} e^{(i)} \\
& =\left(f_{i} / g\right) \sum_{R} a_{R \chi_{V}}(R), \\
& =\left(f_{i} / g\right) \sum_{\rho} x_{V}(\rho) \sum_{R \in \rho} a_{R} .
\end{aligned}
$$

Now one obtains ${ }^{9}$

$$
\sum_{R \in p} a_{R}=\left(g_{p} / f_{i}\right) \chi^{(i)}(\rho)^{*},
$$

where $g_{\rho}$ is the number of elements in the $\rho$ th class. Thus,

$$
t_{V}^{(i)}=(1 / g) \sum_{\rho} g_{p} \chi^{(i)}(\rho) * x_{V}(\rho),
$$

and is just the number of times that the $i$ th irreducible representation appears in the completely reduced form of $D_{V}(R)$.

The form of $e^{(i)}$ may be examined more closely. It was shown above that $\left|a_{R}\right| \leq 1$. The matrix $D^{(\hat{i})}(R)$ for the case $\left|a_{R}\right|=1$ has the form

$$
\left[\begin{array}{cc}
a_{R} & 0 \\
0 & \bar{D}(R)
\end{array}\right],
$$

since $D^{(i)}(R)$ is unitary. Thus the subset of elements of the group for which $\left|a_{R}\right|=1$ all have representation matrices which appear in reduced form. This subset must be a subgroup since inverses, products, and $I$ also will be in reduced form. This result follows easily if the $D^{(i)}(R)$ form a faithful representation of the group. If $D^{(i)}(R)$ is not faithful for the group itself, it certainly is for some quotient group and the result still follows. We denote the subgroup by $G_{s}$, and it is seen that the $a_{R}{ }^{*}\left(R \in G_{s}\right)$ are one-dimensional, irreducible representations of $G_{s}$. We also define $E$ by

$$
E=\sum_{R \in G_{s}} a_{R} R \text {. }
$$

Let $X_{1}(=I), X_{2}, \cdots, X_{k}, k=g / g_{s}$ be a set of right coset generators for $G_{8}$. Hence, we get a factored form of $e^{(i)}$ as

$$
e^{(i)}=f_{i} / g E \sum_{i=1}^{k} a_{X_{i}} X_{i}
$$

There is also a left coset form for $e^{(i)}$ and in fact,

$$
E\left(\sum_{i} a_{X_{i}} X_{i}\right)=\left(\sum_{i} a_{X_{i}} X_{i}\right) E .
$$

The modulus of the subalgebra corresponding to the $i$ th irreducible representation is obtained from the characters of this representation,

$$
m^{(i)}=\left(f_{i} / g\right) \sum_{R} \chi^{(i)}\left(R^{-1}\right) R,
$$

${ }^{9}$ D. E. Littlewood, Rev. Mod. Phys. 39, 56 (1967). 
where the characters satisfy the relation

$$
\chi^{(i)}(R)=\chi^{(i)}\left(R^{-1}\right)^{*}
$$

and hence $m^{(i)}$ is automatically Hermitian. Using the modulus another form of $e^{(i)}$ is possible, viz.,

$$
e^{(i)}=\left(1 / g_{s}\right) E m^{(i)}=\left(1 / g_{s}\right) m^{(i)} E
$$

Thus the structure of the $e^{(i)}$ we have is determined by $G_{\varepsilon}$ and one of its one-dimensional irreducible representations.

The general problem of the determination of an $e^{(i)}$ for any group cannot be said to be solved in a very systematic fashion. For the point groups, geometric considerations can yield the $D^{(i)}(R)$ and hence an $e^{(i)}$. For the symmetric groups considerations of Young tableaux can be used. In general, Eq. (12) appears to offer a convenient method for the determination of $e^{(i)}$ for an arbitrary group if $g$ is not too large. One needs merely to find a subgroup with an

$$
e_{s}^{(i)}=\left(f_{i} / g\right) \sum_{R \in G_{s}} b_{R} R
$$

such that

$$
\left(f_{i} / g_{s}\right) \sum_{R \in G_{s}} \chi^{(j)}(R)^{*} b_{R}=g / f_{j}
$$

where $x^{(j)}(R)$ is the character of the $j$ th irreducible representation of the larger group. When a subgroup and one of its characteristic units satisfying Eq. (13) are found, one obtains

$$
e^{(j)}=e_{8}^{(i)} m^{(j)}=m^{(j)} e_{8}^{(i)}
$$

as a characteristic unit for the larger group. An example of the use of Eqs. (12) and (13) may be found in the Appendix.

It is easily shown that characteristic units $e^{(i)}$ and $e^{(j) \prime}$ corresponding to two groups $G$ and $G^{\prime}$, respectively, give as a characteristic unit for $G \times G^{\prime}$, the cross product, $e^{(i)} \times e^{(j) \prime}$.

\section{THE SYMMETRIC GROUPS}

The symmetric group, $S_{n}$, is defined as the group of all permutations of $n$ objects. As is well known, each irreducible representation of $S_{n}$ is associated with a different partition of $n$, and the representations may be discussed in terms of Young tableaux, ${ }^{10}$ the shapes of which are determined by the partitions.

Two partitions $(\mu)$ and $(\bar{\mu})$ and the corresponding representations are said to be conjugate if the two shapes transform into one another when reflected through a diagonal from the upper left towards the lower right. Certain partitions and their representations are self-conjugate.

Young showed ${ }^{10}$ that a primitive characteristic unit corresponding to $(\mu)$ may be formed by consideration of one tableau with shape $(\mu)$. If we consider those permutations of $S_{n}$ which involve only the numerals in

\footnotetext{
10 See, e.g., D. E. Rutherford, Substitutional Analysis
} (Edinburgh Üniversity Press, London, 1948). one row or column of the tableau, these form a subgroup of $S_{n}$. We form the sum of the permutations from the $i$ th row

$$
P_{i}=\sum \pi_{i}
$$

and the quantity $P_{i} / \mu_{i}$ ! is evidently an idempotent element of the Frobenius algebra of $S_{n}$. If the partition $(\mu)$ has $k$ parts $\mu_{1}, \mu_{2}, \cdots, \mu_{k}$ and $(\bar{\mu})$ has $\bar{k}$ parts $\bar{\mu}_{\overline{1}}$, $\bar{\mu}_{\overline{2}}, \cdots, \bar{\mu}_{\bar{k}}$, the $\bar{j}$ th column of the shape of $(\mu)$ has $\bar{\mu}_{\bar{j}}$ entries. We form the sum

$$
N_{\bar{j}}=\sum \epsilon_{\pi \bar{j}} \pi_{\bar{j}}
$$

where $\epsilon_{\pi j}$ is +1 for even permutations and -1 for odd permutations. The quantity $N_{\bar{j}} / \bar{\mu}_{\bar{j}}$ ! is also an idempotent element of the algebra of $S_{n}$. The characteristic unit is obtained from

with

$$
\theta N_{\mathrm{I}} N_{\overline{2}} \cdots N_{\bar{k}} P_{1} P_{2} \cdots P_{k}=e^{(\mu)},
$$

$$
\theta=f_{(\mu)} / n !
$$

It should be emphasized that $e^{(\mu)}$ in Eq. (16) is not factored in the form of Eq. (10). In fact, Eq. (16) does not define a Hermitian characteristic unit but gives one associated with the natural irreducible representation. We may form a Hermitian $e^{(\mu)}$ by (3):

$$
\theta^{\prime} P_{1} P_{2} \cdots P_{k} N_{\mathrm{I}} N_{2} \cdots N_{\bar{k}} P_{1} P_{2} \cdots P_{k}=e^{(\mu)}=e^{(\mu) \dagger},
$$

where

$$
\theta^{\prime}=f_{(\mu)} /\left(n ! \mu_{1} ! \mu_{2} ! \cdots \mu_{k} !\right)
$$

According to Sec. II there is a unitary representation of $S_{n}, D^{(\mu)}(\pi)$, such that $D^{(\mu)}(\pi)_{11}$ is the coefficient of $\pi$ in $e^{(\mu)}$. Since all the coefficients are real the complex conjugate is not needed here.

Littlewood has defined Young tableaux with repeated elements. ${ }^{11}$ If $m$ symbols, $a_{1}, a_{2}, \cdots, a_{m}$, with $a_{i}$ occurring $\lambda_{i}$ times and $\sum_{i} \lambda_{i}=n$ are defined and placed in a shape corresponding to a partition of $n$, such a tableau is obtained. The $a_{i}$ are assumed to be ordered, $a_{1}<a_{2}<\cdots<a_{m}$ and standard tableaux with repeated elements are those for which the symbols in a row are nondecreasing toward the right and definitely increasing down a column. This concept will be used later in computing the trace of $e^{(\mu)}$ with respect to a particular basis.

\section{THE REPRESENTATIONS OF $S_{n}$ INDUCED BY A CERTAIN VECTOR SPACE}

We assume we have a vector space of finite dimension $m, V$, and an orthonormal basis $v_{1}, v_{2}, \cdots, v_{m}$, thus $\left\langle v_{i} \mid v_{j}\right\rangle=\delta_{i j}$. Corresponding to $S_{n}(n \geq m)$ we may form the cross product space $V_{n}=V(1) \times \bar{V}(2) \times \cdots \times V(n)$. Basis elements of $V_{n}$ will be written

$$
\left|v_{i_{1}}(1) v_{i_{2}}(2) \cdots v_{i_{n}}(n)\right\rangle=|(i)\rangle
$$

where the $i_{j}$ cannot all be different unless $m=n$. An element of $S_{n}, \pi$, operates on $|(i)\rangle$ by permuting the subscripts on the $v$.

\footnotetext{
${ }^{11}$ D. E. Littlewood, Ref. 10, p. 80.
} 
$V_{n}$ is decomposible with respect to $S_{n}$. The linear subspace of $V_{n}$ spanned by those basis vectors of $V_{n}$ such that $v_{1}$ occurs $\mu_{1}$ times, $v_{2}$ occurs $\lambda_{2}$ times, $\cdots, v_{m}$ occurs $\mu_{m}$ times is an invariant subspace with respect to $S_{n} .(\lambda)=\left(\lambda_{1}, \lambda_{2}, \cdots, \lambda_{m}\right)$ is an ordered partition of $n$ with $m$ parts, and this subspace has a dimensionality of $n ! /\left(\lambda_{1} ! \lambda_{2} ! \cdots \lambda_{n} !\right)$. The subspace denoted is $V_{n}(\lambda)$.

Littlewood ${ }^{11}$ proves a theorem which is equivalent to the theorem that the trace of $e^{(\mu)}$ with respect to $V_{n}(\lambda)$ is equal to the number of standard tableaux with repeated elements which can be constructed from $\lambda_{1}$ symbols $v_{1}, \lambda_{2}$ symbols $v_{2}$, etc., where the ordering is arranged so that $\lambda_{1} \geq \lambda_{2} \geq \cdots \geq \lambda_{m}$ and $v_{1}<v_{2}<\cdots<v_{m}$.

As an example we may take $(\mu)=\left(2^{2}, 1\right)$ and $(\lambda)=$ $\left(2,1^{3}\right)$. Using the symbols $1,1,2,3,4$ corresponding to $(\lambda)$ we have

$\begin{array}{lllll}1 & 1 & & 1 & 1 \\ 2 & 3 & & 2 & 4 \\ 4 & & & 3 & \end{array}$

and the trace $t_{(\lambda)}{ }^{(\mu)}=2$. It is easily seen that the $t_{(\lambda)}{ }^{(\mu)}=0$ if $(\lambda)$ has fewer parts than $(\mu)$ and that $t_{(\mu)}{ }^{(\mu)}=1$. If $(\lambda)=\left(1^{n}\right)$ the representation provided by $V_{n}\left(1^{n}\right)$ is evidently the regular representation of $S_{n}$ and $t_{\left.(1)^{n}\right)}{ }^{(\mu)}=f_{(\mu)}$. A similar result is given by Goddard. ${ }^{5}$

\section{CONSTRUCTION OF SPIN EIGENFUNCTIONS}

There is a well-known connection between vectors of $V_{n}$ which provide bases for irreducible representations of $S_{n}$ and vectors of $V_{n}$ which provide bases for irreducible representations of some other group which operates in $V .{ }^{12}$ Explicitly, it is assumed we have a system containing $n$ electrons and we examine a state where $n-k$ particles have spin functions $\alpha$ and $k$ particles have spin functions $\beta$. We may apply the results of Sec. IV, and in this case $m=2$ and $(\lambda)=$ $(n-k, k)$. An essential result here is that $t_{(\lambda)}^{(\mu)}=0$ for all partitions $(\mu)$ containing more than two parts and also $(\mu)=(n-l, l)$ with $l>k \cdot t_{(\lambda)}{ }^{(\mu)}=1$ for all other $(\mu)$. For example, with $(\lambda)=(4,1)$ we have only the tableaux:

$$
\begin{array}{ll}
\mu=(5) & \mu=(4,1) \\
\alpha \alpha \alpha \alpha \beta \beta & \alpha \alpha \alpha \alpha \\
& \beta
\end{array}
$$

This result was proved by Wigner ${ }^{13}$ by direct analysis of the representation provided by $V_{n}$. If $n-k<k$ we obtain the same result by the ordering $\beta<\alpha$. The other result quoted in this section is that the function

$$
e^{(\mu)}|\alpha(1) \cdots \alpha(n-k) \beta(n-k+1) \cdots \beta(n)\rangle
$$

is an eigenfunction of the total spin angular momentum operator with eigenvalue $\hbar^{2}\left(\frac{1}{2} n-l\right)\left(\frac{1}{2} n-l+1\right), l \leq k$ and, of course, the construction indicates the eigenvalue of $S_{z}$ to be $\hbar\left(\frac{1}{2} n-k\right)$.

\footnotetext{
12 F. D. Murnaghan, The Theory of Group Representations (Johns Hopkins Press, Baltimore, 1938).

${ }^{13}$ E, P. Wigner, Ref. 12.
}

\section{ANTISYMMETRIC EIGENFUNCTIONS OF THE SPIN}

Wigner $^{13}$ also proved that an antisymmetric eigenfunction of the spin involving both spatial and spin functions may be constructed from basis functions corresponding to conjugate partitions of $S_{n}$,

$$
|\Psi(\lambda)\rangle=\sum_{l=1}^{f(\lambda)} u_{l}^{(\bar{\mu})} A_{l}^{(\mu)},
$$

where $u_{l}^{(\bar{\mu})}$ is a spatial function and $A_{l}{ }^{(\mu)}$ is the spin function

$$
\begin{aligned}
e_{l 1}{ }^{(\mu)}|\alpha(1) \cdots \alpha(n-k) \beta(n-k+1) \cdots \beta(n)\rangle & \\
& =e_{l 1}^{(\mu)}|(\mu)\rangle .
\end{aligned}
$$

The spatial functions are constructed from $V_{n}\left(\lambda^{\prime}\right)$ involving spatial orbitals with the use of $e_{l 1}{ }^{(\bar{\mu})}$. The restrictions on $(\lambda)$ for which $A_{l}^{(\mu)}$ exist impose similar restrictions on the $(\bar{\mu})$ which need to be considered. Actually, Eq. (18) is correct only when $(\mu) \neq(\bar{\mu})$. There are two cases, $n=3,4$ when two-part partitions are self-conjugate and in both these cases $f_{(\mu)}=2$ and Eq. (18) takes the form

$$
|\psi(\mu)\rangle=u_{1}^{(\mu)} A_{2}^{(\mu)}-u_{2}{ }^{(\mu)} A_{1}{ }^{(\mu)} .
$$

Since the only $(\mu)$ occurring have $\leq$ two parts the only shapes $(\bar{\mu})$ occurring have one or two columns. It is also evident that two antisymmetric functions $|\Psi(\mu)\rangle$ and $|\Phi(\mu)\rangle$ can differ in the spatial part only since the trace of $e^{(\mu)}$ with respect to the product space of the spins is 1 .

We wish to calculate the inner product of $|\Phi(\mu)\rangle$ and $Q|\Psi(\mu)\rangle$ where $Q$ is a Hermitian, spin-free operator.

$$
\langle\Phi(\mu)|Q| \Psi(\mu)\rangle=\sum_{l=1}^{f(\mu)}\left\langle u_{l}^{(\bar{\mu})}|Q| u_{l}^{(\bar{\mu})}\right\rangle\left\langle A_{l}^{(\mu)} \mid A_{l}^{(\mu)}\right\rangle .
$$

If it is assumed that the spin functions are normalized and if Eq. (6) is used, it is easily shown that ${ }^{8}$

$$
\langle\Phi(\mu)|Q| \Psi(\mu)\rangle=f_{(\lambda)}\left\langle u_{1}^{(\bar{\mu}) '}|Q| u_{1}^{(\bar{\mu})}\right\rangle .
$$

If now it is assumed that the spatial functions are in the space $V_{n}(\lambda)$

$$
\begin{aligned}
\left|u_{1}^{(\bar{\mu})}\right\rangle & =e^{(\mu)}|u(\lambda)\rangle, \\
\left|u_{1}^{(\bar{\mu}) \prime}\right\rangle & =e^{(\mu)}\left|u^{\prime}(\lambda)\right\rangle,
\end{aligned}
$$

and Eq. (20) becomes

$$
\langle\Phi(\mu)|Q| \Psi(\mu)\rangle=f_{(\mu)}\left\langle u^{\prime}(\lambda)\left|Q e^{(\mu)}\right| u(\lambda)\right\rangle,
$$

which is the final result. Thus, as was emphasized by Matsen, ${ }^{3}$ all reference to the spin functions has disappeared in this case of a spin-free operator, $Q$. In addition, it is seen that we need only one characteristic unit corresponding to the representation $(\bar{\mu})$, for the case of the spin corresponding to $(\mu)$. 


\section{PROJECTED HARTREE PRODUCT WAVEFUNCTIONS}

We may now return to the problem outlined in the Introduction and state it in a slightly different form.

In the following, it is assumed that a system of $n$ electrons in a spin state $S$ is under investigation. Of course, $S$ is restricted

according as $n$ is

$$
n / 2 \geq S \geq\left\{0^{1 / 2}\right.
$$

$$
\left\{\begin{array}{l}
\text { odd } \\
\text { even }
\end{array},\right.
$$

and the partition of $n$ for $S$ is $(\mu)=[(n / 2)+S$, $(n / 2)-S]$ and $(\bar{\mu})=\left(2^{(n / 2)-S}, 1^{2 S}\right)$.

We inquire as to a best set of $m$ one-electron functions for describing the lowest spin $S$ energy of the system. The number of functions $m$ is restricted below by $m \geq(n / 2)+S$ as we shall see. It was mentioned in the Introduction that the method under consideration is an extension of the independent particle model so we arbitrarily assume $m \leq n$. The criterion used is, of course, a minimum in $W$,

$$
W=\left\langle u(\lambda)\left|H e^{(i)}\right| u(\lambda)\right\rangle /\left\langle u(\lambda)\left|e^{(i)}\right| u(\lambda)\right\rangle,
$$

where $H$ is a spin-free Hamiltonian for the system, $e^{(i)}$ is a characteristic unit of the cross product group of spatial and exchange symmetries, and $|u(\lambda)\rangle$ stands for a simple product wavefunction made from the $m$ functions $u_{1}, u_{2}, \cdots, u_{m}$, with $(\lambda)$ being a partition of $n$ of $m$ parts such that $u_{i}$ occurs $\lambda_{i}$ times in the $|u(\lambda)\rangle$,

$$
\begin{aligned}
|u(\lambda)\rangle=u_{1}(1) u_{1}(2) & \cdots \\
& \times u_{1}\left(\lambda_{1}\right) \\
& \times u_{2}\left(\lambda_{1}+1\right) \cdots u_{2}\left(\lambda_{1}+\lambda_{2}\right) \\
& \times \cdots \\
& \times u_{m}\left(n+1-\lambda_{m}\right) \cdots u_{m}(n) .
\end{aligned}
$$

Since the $e^{(i)}=e^{(k)} \times e^{(\bar{\mu})}$ and $(\bar{\mu})$ has a shape consisting of one or two columns no function can appear more than twice in Eq. (23). Thus $(\lambda)$ must be a partition, $\left(2^{n-m}, 1^{2 m-n}\right)$ and, since $(\bar{\mu})=\left(2^{(n / 2)-S}, 1^{2 S}\right)$ there results, $m \geq(n / 2)+S$, because otherwise standard tableaux with repeated elements of shape $(\bar{\mu})$ cannot be constructed.

Actually, the result of minimizing Eq. (22) is not in general independent of the detailed form of $e^{(i)}$. We may remove this dependence and still retain the spirit if not the details of this method by using instead of $|u(\lambda)\rangle$ a linear combination of product functions, each one a permutation of the same $m$ spatial functions, i.e., instead of Eq. (23)

$$
|u(\lambda)\rangle=\sum_{i=1}^{t\left(\gamma^{\gamma}\right)} \gamma_{i} \pi_{i}\left[u_{1}(1) \cdots u_{m}(n)\right],
$$

where $\pi_{i}$ are certain selected permutations from $S_{n}$, and the number of terms is, as the upper limit indicates, equal to trace of $e^{(\bar{\mu})}$ with respect to $V_{n}(\lambda)$. The function of Eq. (23a) yields results equivalent to that of $\mathrm{Eq}$. (28) in Ref. 3a. The vectors $e^{(\bar{\mu})} \pi_{i} u(1) \cdots u_{m}(n)$; $i=1, \cdots, t_{(\lambda)}^{(\bar{\mu})}$ span a linear subspace of $V_{n}(\mu)$ which is invariant with respect to extrema of $W$ with variations in the $\gamma_{i}$, although this subspace is not invariant under the operations of $S_{n}$. In order to choose the $\pi_{i}$ in Eq. (23a), the factored form of $e^{(\vec{\mu})}$ as shown in Eq. (10) may be utilized, and we need choose from the set of right coset generators.

In this new variational problem we look for extrema of $W$ with respect to variations in the $\gamma_{i}$ as well as variations in the functions $u_{l}$. Although we are no longer projecting a single product function, we still can say that the projected wavefunction is determined by $m$ one-particle space functions and hence is based on an independent particle scheme. In those cases where $t_{(\lambda)}{ }^{(\vec{\mu})}=1$ there is no difference between the two problems, of course. The cases where $t_{\lambda}(\bar{\mu})=1$ are of two types. The first case is when $(\lambda)=(\bar{\mu})$ and $|\Psi\rangle$ as defined by Eq. (18) or $\left(18^{\prime}\right)$ is equivalent to the single Slater determinant constructed from the same spatial functions and appropriate spin functions. The other case where $t_{(\lambda)}^{(\bar{\mu})}=1$ occurs when $n=$ even, $(\bar{\mu})=\left(2^{n / 2}\right)$, and $(\lambda)=\left(2^{(n / 2)-1}, 1^{2}\right)$, and $|\Psi\rangle$ is equivalent to the sum of exactly two Slater determinants.

The solution of Eq. (22) for the minimum $W$ with the $|u(\lambda)\rangle$ of Eq. $\left(23^{\prime}\right)$ is expected to yield the lowest value when $m=n$, since there are then the fewest additional restrictions. It is not known, however, whether useful results may be obtained when $m<n$, i.e., whether these restrictions cause serious changes in the calculated energy and in the values of other quantities that might be calculated from the wavefunctions as compared to the most general case when $m=n$. Of course, the special case $m=n / 2, n$ even, is equivalent to the restricted Hartree-Fock solution.

\section{AN ITERATIVE SOLUTION}

If the first variation of $W$ in Eq. (22) is computed and $\delta W=0$, a set of coupled integro-differential equations is obtained which in general are very difficult to solve. In fact, as pointed out in the Introduction, they possess no unique solution, in general. This approach to the minimization of $W$ will not be discussed in this article.

It appears that a much more profitable path is to use the technique devised by Roothaan ${ }^{14}$ which has been used so effectively for the restricted Hartree-Fock equations (see Ref. 5 also). Thus, it is assumed, that we have a finite set of functions $v_{j}$ and we write

$$
u_{k}=\sum_{j=1}^{M} a_{k j} v_{j}
$$

The number of functions $M$ must be greater than or equal to $m$. Using Eqs. (24), $\left(23^{\prime}\right)$, and (22) it is seen that $W$ is a rational homogeneous function of the variables $\gamma_{i}$ and $a_{k j}$. The degree of the homogeneity is zero. The total degree of the homogeneous polynomials in the numerator and denominator is $2(n+1)$. If $u_{k}$ is present $\lambda_{k}(=1$ or 2$)$ times in Eq. (23a) the degree of homogeneity of either numerator or denominator with

${ }^{14}$ C. C. J. Roothaan, Rev. Mod. Phys. 23, 69 (1951). 
respect to $a_{k j} ; j=1,2, \cdots, M$ is $2 \lambda_{k}$. These polynomials are of degree 2 with respect to the $\gamma_{i}$. Let us choose $(m-1) M$ constants $c_{k j} ; j=1,2, \cdots, M ; k=1, \cdots, m$ except $k \neq i$ and $t_{(\lambda)}{ }^{(\bar{\mu})}$ constants $\xi_{l}$ and let

and

$$
a_{k j}=c_{k j} ; \quad k \neq i
$$

$$
\gamma_{l}=\xi_{l} \text {. }
$$

The minimization of $W$ with respect to the remaining $a_{i j}$ is just a standard matrix diagonalization with nonzero overlap if $\lambda_{i}=1$ or a minimization of the same type as occurs in Roothaan's method if $\lambda_{i}=2$. If instead the restrictions (26) are removed the former type of problem is obtained. An iterative procedure obtained by setting

$$
c_{i j}{ }^{(p+1)}=a_{i j},
$$

with $a_{i j}$ the solution of $\delta W=0$ for

$$
\begin{aligned}
a_{k j} & =c_{k j}{ }^{(p+1)} ; & & k<i, \\
& =c_{k j}{ }^{(p)} ; & & k>i, \\
\gamma_{l} & =\xi_{l}{ }^{(p)}, & &
\end{aligned}
$$

and a choice for $c_{k j}{ }^{(0)}, \xi_{l}{ }^{(0)}$ will converge for a least some choices of the starting vectors. Alternatively, a simple binary search for the minimum is possible on a computer, but this method is very slow.

\section{DISCUSSION}

The projected Hartree product procedure discussed in this article is a type of restricted configuration interaction calculation and its connection with some other types of treatments of the simple system $\mathrm{H}_{2}$ will be discussed.

The singlet state of any two spin system is associated with $(\mu)=\left(\mathbf{1}^{2}\right)$ and hence $(\boldsymbol{\mu})=(2)$. The state in which we are interested is ${ }^{1} \Sigma_{g}+$, thus examination of the $D_{\infty h}$ group gives us for the appropriate characteristic unit for $D_{\infty h} \times S_{2}$

$$
\begin{aligned}
e\left({ }^{1} \Sigma_{g}+\right)=(16 \pi)^{-1} & {[I+(12)] } \\
& \times \int_{0}^{2 \pi}\left[c_{\phi}+\sigma(\phi)+S_{\phi}+c_{2}(\phi)\right] d_{\phi} .
\end{aligned}
$$

If this projection is to be applied to a cylindrically symmetric function, the integrals in Eq. (27) may be evaluated beforehand and with this restriction

$$
e\left({ }^{1} \Sigma_{g}+\right)^{\prime}=\frac{1}{4}[I+(12)]\left[I+\sigma_{h}\right],
$$

may be used.

We have two cases for $(\lambda)$ to examine, (2) and $\left(1^{2}\right)$. For each of these cases $t_{(\lambda)}(\bar{\mu})=1$.

$$
\text { A. }(\lambda)=(2)
$$

Here $|u(\lambda)\rangle=u(1) u(2)$, and to make a connection with some of the previous calculations of the structure of $\mathrm{H}_{2}$, it is assumed that the basis set $v_{j}$ in Eq. (24) consists of the two $1 S$ functions located at the two nuclei. Thus one obtains

$$
\begin{aligned}
u & =a\left(1 S_{a}\right)+b\left(1 S_{b}\right), \\
e\left({ }^{\prime} \Sigma_{o}+\right)|u(2)\rangle & =B\left(1 S_{a}+1 S_{b}\right)(1)\left(1 S_{a}+1 S_{b}\right)(2),
\end{aligned}
$$

and $B=(a+b)^{2} / 2$, and the result is the simple antisymmetrized molecular orbital wavefunction for hydrogen. ${ }^{15}$ It is obvious that $a$ and $b$ are undetermined by $\delta W=0$ in this case, and a unique result for $u$ is not obtained as was mentioned above. Although this is a rather trivial case, the added restriction of $a=\gamma b, \gamma$ any constant, yields a unique result for $|u(2)\rangle$ without losing generality.

$$
\text { B. }(\lambda)=\left(1^{2}\right)
$$

If we use the same basis set $v_{j}$ as above, we obtain in this case

$$
\left|u\left(1^{2}\right)\right\rangle=\left[a 1 S_{a}(1)+b 1 S_{b}(1)\right]\left[c 1 S_{a}(2)+d 1 S_{b}(2)\right],
$$

and

$$
\begin{aligned}
& \begin{array}{l}
e\left(\Sigma^{+}\right)^{\prime}\left|u\left(1^{2}\right)\right\rangle=A\left[1 S_{a}(1) 1 S_{b}(2)+1 S_{a}(2) 1 S_{b}(1)\right] \\
+B\left[1 S_{a}(1) 1 S_{a}(2)+1 S_{b}(1)+1 S_{b}(2)\right],
\end{array} \\
& \text { where } \begin{aligned}
\text { and } & A=(a d+b c) / 2, \\
& B=(a c+b d) / 2 .
\end{aligned}
\end{aligned}
$$

Here again $\left|u\left(1^{2}\right)\right\rangle$ is not uniquely determined by $\delta W=0$ but the added restrictions

$$
b=c, \quad a=d,
$$

for example, make $\left|u\left(1^{2}\right)\right\rangle$ a unique ray in the Hilbert space. The wavefunction defined in Eq. (28) is, of course, the Heitler-London function with the "ionic" term added. ${ }^{15}$ The present treatment with the restrictions of Eq. (29) is identical with that of Coulson and Fischer ${ }^{16}$ and is equivalent to the AMO treatment for $\mathrm{H}_{2}$, and thus is capable of some correlation of the type called right and left by Lennard-Jones and Pople. ${ }^{17}$

If more basis functions are added to Eq. (24), one obtains results equivalent or nearly equivalent to other more extended treatments of $\mathrm{H}_{2}$. A study of the $\mathrm{H}_{2}$ molecule with a more extended basis set is nearly finished and the results will be reported shortly.

As was emphasized above and elsewhere ${ }^{2}$ the results obtained by the PHF method and the one here under discussion are extensions of the independent particle model for a system and yield a set of one particle spatial functions which with an appropriate projection operator yield a unique configuration interaction function for the system. The importance of this lies in the ease with which qualitative reasoning can be applied to single orbitals as compared with complicated multiparticle functions. However, many other cases need to be studied to determine the behavior of orbitals determined in this way. The converse procedure can be utilized, also. The eigenfunction for a system determined by a configuration interaction calculation with a

\footnotetext{
15 See, e.g., J. C. Slater, Quantum Theory of Molecules and Solids (McGraw-Hill Book Co., New York, 1963), Vol. 1.

${ }_{16}$ C. A. Coulson and I. Fischer, Phil. Mag. 40, 386 (1949).

${ }^{17} \mathrm{~J}$. D. Lennard-Jones and J. A. Pople, Phil. Mag. 43, 581 (1952).
} 
TABLE I. Calculated results for $\mathrm{He}$ (see Sec. X).

\begin{tabular}{ccccccc}
\hline$z$ & $\alpha_{1}$ & $\alpha_{2}$ & $\beta_{1}$ & $\beta_{2}$ & $a$ & $E$ \\
\hline 2 & 1.202 & 2.149 & 2.345 & 1.563 & 0.193 & -2.8806 \\
3 & 2.083 & 3.286 & 2.311 & 2.303 & 0.226 & -7.2480 \\
4 & 2.987 & 4.384 & 3.491 & 3.487 & 0.182 & -13.625 \\
5 & 3.911 & 5.463 & 4.669 & 4.605 & 0.154 & -22.001 \\
6 & 4.829 & 6.546 & 5.304 & 5.415 & 0.148 & -32.374 \\
\hline
\end{tabular}

spin-free Hamiltonian can be factored (in general approximately) into a Hartree product function which will, with the projection operator, reproduce an approximate form of the configuration interaction wavefunction.

\section{A SIMPLE Calculation OF $\mathrm{He}$}

A simple calculation of the ground-state energy of the helium atom has been carried out using the projected Hartree product method in the form discussed in this article. We investigate the results of using what are essentially two $s p$ hybrid functions for the two spatial orbitals. Since $t_{\left(1^{2}\right)}{ }^{(2)}=1$ in this case Eq. (23) may be utilized and we have

$$
\left|u\left(1^{2}\right)\right\rangle=\left[S_{1}(1)+a p_{1}(1)\right]\left[S_{2}(2)-a p_{2}(2)\right],
$$

where

$$
\begin{aligned}
& S_{i}=c_{i} \exp \left(-\alpha_{i} r\right), \\
& p_{i}=c_{i}{ }^{\prime} z \exp \left(-\beta_{i} r\right),
\end{aligned}
$$

and we have set the coefficients of the $p$-type functions equal and opposite in sign to insure a unique result. This does not decrease the generality of the result. The integrals involved here are, of course, all well-known in analytic form and the expression for $W$ is easily written out as a function of $\alpha_{1}, \alpha_{2}, \beta_{1}, \beta_{2}, a$, and $Z$, the atomic number of the nucleus. Although the energy is not very good, an interesting case is that obtained by taking $\alpha_{1}=\alpha_{2}=\beta_{1}=\beta_{2}=\alpha$ since the result may be worked out analytically. The characteristic unit for a ${ }^{1} S$ state is

$$
e\left({ }^{1} S\right)=(2 V)^{-1}[I+(12)] \int R d \tau,
$$

where $R$ is an element of the three-dimensional rotation group and $V$ is the volume of this group. Identical results are obtained, of course, if the integral in Eq. (30) is replaced by any projection operator singling out an $S$ state.

With the Hamiltonian

$$
H=-\frac{1}{2}\left(\nabla_{1}^{2}+\nabla_{2}^{2}\right)-Z\left[\left(1 / r_{1}\right)+\left(1 / r_{2}\right)\right]+\left(1 / r_{12}\right)
$$

and the restrictions on the exponential scale factors noted above, the minimum value of $W$ is

$$
\begin{aligned}
& W=-\alpha^{2}, \\
& \alpha=\frac{(1536 z-480)+112 a^{2}+(256 z-111) a^{4}}{\left(1536+512 a^{4}\right)}, \\
& a= \pm\left\{\frac{112}{(256 z-49)+\left[(256 z-49)^{2}+12544 / 3\right]^{1 / 2}}\right\}^{1 / 2},
\end{aligned}
$$

in atomic units. For $z=2, W=-2.8625$, a result only somewhat better than the simple screening constant treatment of He. In this special case it is easily shown that

$$
\begin{aligned}
e\left({ }^{1} S\right) \mid & \left.u\left(1^{2}\right)\right\rangle \\
& =(\text { const })\left[1-\mu\left(r_{1}^{2}+r_{2}^{2}-r_{12}^{2}\right)\right] \exp \left[-\alpha\left(r_{1}+r_{2}\right)\right],
\end{aligned}
$$

where $\mu=$ (const) $a^{2}$. Thus this treatment contains some in and out as well as angular correlation, but with forced common coefficients. Also, in this case $a=0.35$, so there is considerable mixing of the $p$-type function with the $s$-type in the orbitals.

Table I gives the results of this treatment as a function of $z$, for the case where $\alpha_{1}, \alpha_{2}, \beta_{1}, \beta_{2}$ are all allowed to have their best values. As can be seen, the amount of $p$ character in the orbitals is considerably less than when $\alpha_{1}=\alpha_{2}=\beta_{1}=\beta_{2}$ is forced. This seems reasonable since $a$ controls both in and out and angular correlation and here the differing exponential scale factors take care of some of the former type of correlation. Also, the inclusion of a large number of nonlinear parameters such as $\alpha_{1}, \alpha_{2}, \beta_{1}, \beta_{2}$ in a variation calculation can lead to an expression for $W$ which is multimodal, and there may be more than one set of nonequivalent minima which have nearly the same values. The values of the parameters in Table I seem to indicate that the lowest minimum slips from one equivalent set to another for $Z$ between the values of 2 and 3 .

These results for He show very clearly the effect of orbitals which lack the symmetry of the total system, and the ability of such orbitals to reproduce electron correlation when used with projection operators. A similar statement can be made for the $\mathrm{H}_{2}$ molecule treatment in Sec. IX.B (but not IX.A). This phenomenon has been discussed with regard to the $\mathrm{H}_{2}$ molecule by Goddard ${ }^{5}$ also.

\section{THE ALLYL RADICAL}

A three-electron system in a doublet state is the simplest that can have a $t_{(\lambda)}^{(\bar{\mu})}>1$. In particular, we have $t_{\left(1^{3}\right)}{ }^{(2,1)}=f_{(2,1)}=2$ for this case, and there are two terms in Eq. $\left(23^{\prime}\right)$. If we apply these results to the allyl radical it is found that the "full" configuration interaction calculation for the $\pi$ system based on three $p$-type atomic orbitals can be transcribed exactly into the form of Eq. $\left(23^{\prime}\right)$. Hirst and Linnet ${ }^{18}$ have given the CI wavefunction for this system and a fairly simple calculation shows that the function

$$
\begin{aligned}
& u_{1}^{(2,1)}=e^{(2,1)}[I+0.233(13)]\{[a(1)-c(1)] \\
& \quad \times[a(2)+1.46 b(2)+c(2)][a(3)+1.68 b(3)+c(3)]\},
\end{aligned}
$$

is equivalent to it. In Eq. (31) we have followed

${ }^{18}$ D. M. Hirst and J. W. Linnett, J. Chem. Soc. 1962, 1035. 
Linnet's notation for the atomic orbitals and

$$
\begin{aligned}
e^{(2,1)} & =\frac{1}{6}[I+(12)][I-(13)][I+(12)], \\
& =\frac{1}{3}[I+(12)]\left[I-\frac{1}{2}(13)-\frac{1}{2}(23)\right] .
\end{aligned}
$$

Although in this case the projected Hartree product function and the "full" CI wavefunction are equivalent, this is not so in general.

\section{APPENDIX: SOME YOUNG OPERATORS}

As an example of the use of Eq. (12) for the construction of primitive characteristic units of a group

TABLE II. Idempotent elements of $S_{4}$ corresponding to the cyclic subgroup of order 4.

$$
\begin{aligned}
& e=\frac{1}{4}[\mathrm{I}+(1234)+(13)(24)+(1432)] \\
& f=\frac{1}{4}[\mathrm{I}+i(1234)-(13)(24)-i(1432)] \\
& g=\frac{1}{4}[\mathrm{I}-i(1234)-(13)(24)+i(1432)] \\
& h=\frac{1}{4}[\mathrm{I}-(1234)+(13)(24)-(1432)]
\end{aligned}
$$

let us consider $S_{4}$. Let us arbitrarily consider the cyclic subgroup of $S_{4}$ made up of $I, \pi[=(1234)], \pi^{2}, \pi^{3}$. Table II shows the idempotent elements of the group algebra of $S_{4}$ corresponding to each of the four irreducible representations of the subgroup. If we now take the modulus of the subalgebra of $S_{4}$ corresponding to $(3,1), m(3,1)$, and multiply in turn by $e, f, g, h$ and then factor according to Eq. (10) one obtains the results given in Table III. As can be seen the coefficient of $I$ in each of the operators is $f_{(3,1)} / 4 !=1 / 8$, hence each is of rank 1 and hence primitive. They are mutually noninteracting projections since

$$
f g=f h=g h=0 .
$$

These three operators can be taken to be $e_{11}^{(3,1)}, e_{22}^{(3,1)}$, and $e_{33}{ }^{(3,1)}$, and by changing the sign of the coefficients of odd permutations one can obtain $e_{i i^{\left(21^{2}\right)}}, i=1,2,3$. As can be seen from $h m(3,1)$ the subgroup of the factored form of $e^{(\mu)}$ is not always identical with the subgroup generating it.

Table IV gives a form for $e^{(\mu)}$ for each of the two column partitions of $S_{4}$ based on Eqs. (17) and (10).

TABle III. Primitive characteristic units for $(3,1)$ of $S_{4}$.

$$
\begin{aligned}
& e m(3,1)=0 \\
& f m(3,1)=[I+i(1234)-(13)(24)-i(1432)]\left[I+\frac{1}{2}(23)+\frac{1}{2}(14)+\frac{1}{2}(12)+\frac{1}{2}(34)\right] / 8 \\
& g m(3,1)=[I-i(1234)-(13)(24)+i(1432)]\left[I+\frac{1}{2}(23)+\frac{1}{2}(14)+\frac{1}{2}(12)+\frac{1}{2}(34)\right] / 8 \\
& h m(3,1)=[I-(1234)+(13)(24)-(1432)+(13)+(24)-(14)(23)-(12)(34)] / 8
\end{aligned}
$$

TABLE IV. $e^{(\mu)}$ for two column partitions of $S_{4}$.

$\left(2,1^{2}\right): \quad e^{\left(2,1^{2}\right)}=[I+(14)-(23)-(14)(23)]\left[I-\frac{1}{2}(12)-\frac{1}{2}(13)-\frac{1}{2}(24)-\frac{1}{2}(34)\right] / 8$

$\left(2^{2}\right): \quad e^{(22)}=[I+(13)+(24)+(13)(24)+(12)(34)+(13)(24)+(1432)+(1234)] \times\left[I-\frac{1}{2}(12)-\frac{1}{2}(13)\right] / 12$ 